\title{
Physical meaning of Lagrange multipliers
}

\author{
Hasan Karabulut \\ Rize University, \\ Faculty of Arts and Sciences, \\ Physics department, \\ 53100 Rize/TURKEY

\begin{abstract}
A rule to assign physical meaning to Lagrange multipliers is discussed. Examples
\end{abstract} \\ from mechanics, statistical mechanics and quantum mechanics are given.
}

\section{INTRODUCTION}

Lagrange multipliers arise frequently in physics, engineering, economics and mathematics in optimization problems with constraints. In statistical mechanics it arises in microcanonical derivation of distribution laws of quantum gases and in some other problems where entropy or free energy is maximized or minimized under the constrains of fixed total particle number and/or energy. In classical mechanics Lagrange multipliers arise in Lagrangian formulation of mechanics with constraints where multipliers are interpreted as constraint forces. In economics and engineering there are plenty of problems that requires optimization of some quantity under some constraints and Lagrange multipliers arise naturally in these fields.

In most of these cases Lagrange multiplier turns out to have a physical meaning at the end of calculation. In economics people seem to have long been aware that the multipliers are related to rate of change of optimized quantity with respect to some parameter in the calculation. In physics it is well known that multipliers usually have precise physical meanings although there is not a general rule to identify their physical meaning directly. In statistical mechanics Lagrange multipliers occurs in many places and the pedagogical articles on them seem to concentrate on how to teach statistical mechanics without using 
Lagrange Multipliers [1, 2, 3, 4, 5] rather than how to find precise physical interpretation of the multipliers. Statistical mechanics textbooks derive physical meaning of multipliers by using supplementary arguments. I have long felt that (at least in statistical mechanics) there must be a direct rule to identify the physical meaning of multipliers and after some research I found that such a rule exist and It works well if the constrains have a well defined physical meaning. It does not seem to work for quantum applications in particular where quantities such as overlap integrals between orbitals do not have a clear physical meaning. But in some areas of physics and engineering as well as in economics the rule works well.

I have come across this rule in economics textbooks and economists have been aware of it for long time. Even the books on mathematical economics written in the sixties and seventies have the rule[6] and there are articles(see Baxley and Moohouse[7] for example) discussing the Lagrange multipliers in economics. Engineers too are interested in Lagrange multipliers and Bertsekas's book [8] on Lagrange multipliers has the above mentioned rule. Traditionally Lagrange multipliers method is introduced in calculus books and they do not discuss physical meaning of multipliers. Even this is changing and two of recent calculus books [9, 10] discuss the meaning of multipliers and present the rule without proof(Actually [10] provides some justification). The Lagrange multipliers are used in constrained variational problems and a proper extension of the rule exists in the literature on calculus of variations. [11]

I have checked the statistical mechanics books that I was able to reach and none of them mentions the rule and I have not come across it elsewhere in the physics literature that I am familiar with. It seems that the rule is not well known in physics community. This prompted me to write this paper and discuss use of this rule in physics. In the paper I will show how the rule makes interpretation of multipliers easy in statistical mechanics and I will also present a quantum application where the rule is not applicable. I also provide some examples that shows how the rule makes interpretation of Lagrange multipliers easy in some mechanics problems.

In the following section I derive the above mentioned rule for discrete variables and I discuss two examples. In the third section I derive the same rule for constrained variational 
problems and I discuss three examples.

\section{DERIVATION OF THE RULE FOR DISCRETE VARIABLES AND EXAMPLES}

First we start by reminding the reader how Lagrange multipliers used in constrained optimization. Suppose we want to find a local maximum or minimum of a function $f\left(x_{1}, x_{2}, \ldots, x_{N}\right)$ under the constraints

$$
g_{i}\left(x_{1}, x_{2}, \ldots, x_{N}\right)=u_{i}, \quad(i=1,2, \ldots, M) .
$$

Lets denote the maximum/minimum point with starred characters $\left(x_{1}^{*}, x_{2}^{*}, \ldots, x_{N}^{*}\right)$ and lets use the compact vector notation $\vec{r}=\left(x_{1}, x_{2}, \ldots, x_{N}\right)$ and $\vec{r}^{*}=\left(x_{1}^{*} x_{2}^{*}, \ldots, x_{N}^{*}\right)$. We also use N-dimensional gradient

$$
\vec{\nabla} f=\frac{\partial f}{\partial x_{1}} \widehat{e}_{1}+\frac{\partial f}{\partial x_{2}} \widehat{e}_{2}+\ldots+\frac{\partial f}{\partial x_{N}} \widehat{e}_{N},
$$

where $\widehat{e}_{1}, \widehat{e}_{2} \ldots \widehat{e}_{N}$ are the unit vectors in the directions $x_{1}, x_{2}, \ldots, x_{N}$. With this notation, at the extremum point we must have

$$
\vec{\nabla} f\left(\vec{r}^{*}\right)=\sum_{i=1}^{M} \lambda_{i} \vec{\nabla} g_{i}\left(\vec{r}^{*}\right),
$$

where $\lambda_{1}, \lambda_{2}, \ldots, \lambda_{M}$ are the Lagrange multipliers. This represents $N$ sets of equations. We also have constraints in eq.(11) which are $M$ sets of equations. We have a total of $N+M$ equations and $N+M$ unknowns $\left(x_{1}^{*}, x_{2}^{*}, \ldots, x_{N}^{*}\right)$ and $\lambda_{1}, \lambda_{2}, \ldots, \lambda_{M}$. and therefore they can be solved in principle. Sometimes this sets of equations are written as

$$
\begin{aligned}
& \partial \Phi / \partial x_{n}=0(n=1,2, \ldots, N), \\
& \partial \Phi / \partial \lambda_{m}=0(m=1,2, \ldots, M) .
\end{aligned}
$$

where $\Phi$ is the auxiliary function

$$
\Phi\left(\vec{r}, \lambda_{1}, \ldots, \lambda_{M}\right)=f(\vec{r})-\sum_{i=1}^{M} \lambda_{i}\left(g_{i}(\vec{r})-u_{i}\right) .
$$


Now we are ready to derive the rule for meaning of the Lagrange multipliers. The coordinate of the extremum point $\vec{r}^{*}$ will be a function of $u_{1}, u_{2}, \ldots, u_{M}$. Therefore the value of $f(\vec{r})$ at the extremum point $\vec{r}^{*}$ will be a function of $u_{1}, u_{2}, \ldots, u_{M}$ too. We denote this function as $F\left(u_{1}, u_{2}, \ldots, u_{M}\right)$ :

$$
F\left(u_{1}, u_{2}, \ldots, u_{M}\right)=f\left(\vec{r}^{*}\right)
$$

Now, suppose each $u_{i}$ changed by an infinitesimal amount: $u_{i} \rightarrow u_{i}+d u_{i}$. This changes the extremum point $\vec{r}^{*}$ by $d \vec{r}^{*}: \vec{r}^{*} \rightarrow \vec{r}^{*}+d \vec{r}^{*}$. The $d u_{i}$ and $d \vec{r}^{*}$ are connected:

$$
d u_{i}=g_{i}\left(\vec{r}^{*}+d \vec{r}^{*}\right)-g_{i}\left(\vec{r}^{*}\right)=\vec{\nabla} g_{i}\left(\vec{r}^{*}\right) \cdot d \vec{r}^{*}
$$

The change in $F$ is

$$
d F=f\left(\vec{r}^{*}+d \vec{r}^{*}\right)-f\left(\vec{r}^{*}\right)=\vec{\nabla} f\left(\vec{r}^{*}\right) \cdot d \vec{r}^{*}
$$

The $\vec{\nabla} f\left(\vec{r}^{*}\right)$ can be written from eq.(3) as

$$
\begin{aligned}
& d F=\sum_{i=1}^{M} \lambda_{i} \cdot \vec{\nabla} g_{i}\left(\vec{r}^{*}\right) \cdot d \vec{r}^{*} \\
& d F=\lambda_{1} d u_{1}+\lambda_{2} d u_{2}+\ldots+\lambda_{M} d u_{M} .
\end{aligned}
$$

This clearly tells us that

$$
\lambda_{i}=\partial F\left(u_{1}, u_{2}, \ldots, u_{M}\right) / \partial u_{i}(i=1,2, \ldots, M)
$$

Usually both $F\left(u_{1}, u_{2}, \ldots, u_{M}\right)$ and $u_{1}, u_{2}, \ldots, u_{M}$ have well defined physical meaning and this relation assigns a physical meaning to the Lagrange multipliers.

\section{A. Example1. Maximum range of a projectile}

It is instructive to do this elementary exercise using Lagrange multiplier. If the components of initial velocity are $V_{x}, V_{y}$ then the flight time of the projectile is $2 V_{y} / g$ and the range is $R\left(V_{x}, V_{y}\right)=2 V_{x} V_{y} / g$. The kinetic energy of the projectile is fixed: $m\left(V_{x}^{2}+V_{y}^{2}\right) / 2=E$. The auxiliary function $\Phi\left(V_{x}, V_{y}, \lambda\right)$ is

$$
\Phi\left(V_{x}, V_{y}, \lambda\right)=2 V_{x} V_{y} / g-\lambda\left[m\left(V_{x}^{2}+V_{y}^{2}\right) / 2-E\right]
$$


Taking partial derivatives with respect to $V_{x}, V_{y}$ and $\lambda$ we get the set of equations

$$
\begin{aligned}
& 2 V_{y} / g-\lambda m V_{x}=0, \\
& 2 V_{x} / g-\lambda m V_{y}=0, \\
& m\left(V_{x}^{2}+V_{y}^{2}\right) / 2=E .
\end{aligned}
$$

The solutions are

$$
\begin{aligned}
V_{x}^{*} & =V_{y}^{*}=\sqrt{E / m}, \\
\lambda & =2 / m g .
\end{aligned}
$$

The maximum range is

$$
R\left(V_{x}^{*}, V_{y}^{*}\right)=(2 / m g) E,
$$

and $\lambda=d R\left(V_{x}^{*}, V_{y}^{*}\right) / d E$ is satisfied. The meaning of $\lambda$ here is the rate of increase of maximum range with energy.

\section{B. Example2. Microcanonical derivation of quantum gas distributions}

Most applications of Lagrange multipliers involve only one multiplier and some involve two multipliers. In physics applications involving more than two multipliers are extremely rare. Here we present a common application in statistical mechanics involving two multipliers. It is the uninteracting quantum (Both Bose and fermi) gases.

We follow Huang's statistical mechanics textbook[12] in the following discussion. We first divide the energy into small intervals $\Delta E_{i}$. In every interval $\Delta E_{i}$ we have $g_{i}$ single particle levels and $n_{i}$ particles occupying these levels. The system size (particle number and volume) is huge such both the $g_{i}$ and $n_{i}$ are large numbers. But the energy intervals $\Delta E_{i}$ are small enough such that we can assume that all $g_{i}$ have the same energy $E_{i}$ where $E_{i}$ is some average energy value within the $\Delta E_{i}$ interval (say the midpoint of the interval). By taking a larger and larger system we can make these assumption more and more accurate. 
The number of different ways of putting $n_{i}$ particles into $g_{i}$ degenerate single particle states for Bose particles is

$$
w_{i}=\frac{\left(n_{i}+g_{i}-1\right) !}{n_{i} !\left(g_{i}-1\right) !}
$$

and for fermi particles is

$$
w_{i}=\frac{g_{i} !}{n_{i} !\left(n_{i}-g_{i}\right) !} .
$$

The total number of ways can be written as

$$
W\left\{n_{i}\right\}=\prod_{i} w_{i}
$$

We want to maximize $W\left\{n_{i}\right\}$ subject to the constraints

$$
\begin{aligned}
\sum_{i} n_{i} & =N \\
\sum_{i} n_{i} E_{i} & =U .
\end{aligned}
$$

Instead of maximizing $W\left\{n_{i}\right\}$ we maximize $k_{B} \ln \left(W\left\{n_{i}\right\}\right)$ for convenience. We write the auxiliary function $\Phi\left(\left\{n_{i}\right\}, \lambda\right)$

$$
\Phi\left(\left\{n_{i}\right\}, \lambda\right)=k_{B} \sum_{i} \ln \left(w_{i}\right)-\lambda_{1}\left(\sum_{i} n_{i} E_{i}-U\right)-\lambda_{2}\left(\sum_{i} n_{i}-N\right),
$$

and use the Stirling approximation to express the factorials in $\ln w_{i}$ and set derivatives $\partial \Phi / \partial n_{i}$ to zero to obtain the equations that $\left\{n_{i}\right\}$ satisfy. When they are solved we obtain

$$
n_{i}^{*}=\frac{g_{i}}{\exp \left(\lambda_{1} E_{i} / k_{B}+\lambda_{2} / k_{B}\right) \pm 1}
$$

where + is for fermions and - is for bosons in the denominator. Here $g_{i}>>1$ is used to obtain this result. The $\lambda_{1}$ and $\lambda_{2}$ is found by solving the equations $\sum_{i} n_{i}^{*}=N$ and $\sum_{i} n_{i}^{*} E_{i}=U$ simultaneously.

Now we have the problem of identifying the thermodynamical meaning of $\lambda_{1}$ and $\lambda_{2}$. Staying within the microcanonical ensemble this identification requires considerable amount of supplementary argument. Of course one can derive eq. (26) using grand canonical ensemble where the quantities appearing in place of $\lambda_{1}$ and $\lambda_{2}$ have fixed meanings in the grand ensemble. But in teaching statistical mechanics we usually want to show that all ensembles are equivalent an we should be able to find physical meaning of $\lambda_{1}$ and $\lambda_{2}$ staying in 
microcanonical ensemble too. Our rule makes this identification a trivial exercise as shown below.

In the microcanonical ensemble the entropy is

$$
S=k_{B} \ln \left(\sum_{\left\{n_{i}\right\}} W\left\{n_{i}\right\}\right),
$$

where the sum runs over all possible $\left\{n_{i}\right\}$ sets. It is shown in statistical mechanics textbooks that in the thermodynamic limit this is equal to $k_{B} \ln W\left\{n_{i}^{*}\right\}$. Therefore $k_{B} \ln W\left\{n_{i}^{*}\right\}$ is the entropy $S(U, N)$ as a function of internal energy $U$ and the particle number $N$. From the rule it follows that the $\lambda_{1}$ and $\lambda_{2}$ are

$$
\begin{aligned}
& \lambda_{1}=(\partial S / \partial U)_{N} \\
& \lambda_{2}=(\partial S / \partial N)_{U} .
\end{aligned}
$$

The first one is easily interpreted as $1 / T$ where $T$ is the temperature of the system. In order to interpret the second one we use the well-known relation

$$
(\partial S / \partial N)_{U} \cdot(\partial N / \partial U)_{S} \cdot(\partial U / \partial S)_{N}=-1 .
$$

From thermodynamics $(\partial U / \partial S)_{N}=T$ and $(\partial N / \partial U)_{S}=\mu^{-1}$ where $\mu$ is the chemical potential of the system. Then we obtain $\lambda_{2}=(\partial S / \partial N)_{U}=-\mu / T$.

As can be seen from this example, in statistical mechanical applications the rule makes supplementary arguments to interpret multipliers unnecessary. I invite the reader to try to find another argument as short as this one (staying within microcanonical ensemble) without using the rule. In statistical mechanics (unlike quantum mechanics for example) both constrained and optimized quantities always have well defined physical meanings and identification of thermodynamical meaning of the multipliers is only one-line argument using this rule. Once the rule is introduced beforehand (in an appendix of a book or as a handout in a class for example) identification of multipliers will be a very precise and concise argument. 


\section{DERIVATION OF THE RULE IN VARIATIONAL PROBLEMS AND EXAMPLES}

We will not discuss the theory of variational calculus here or how the Lagrange multipliers are used in variational calculus. We will merely state basic results and derive the equivalent formula for the interpretation of Lagrange multipliers.

In the simplest case of variational problems we are trying to find the function $y(x)$ that makes the integral

$$
I[y]=\int_{a}^{b} f\left(x, y, y^{\prime}\right) d x,
$$

an extremum where $y^{\prime}=d y / d x$ and $y(a)$ and $y(b)$ are fixed. The function $y^{*}$ that makes $I[y]$ an extremum satisfies the Euler differential equation

$$
\frac{\delta I\left[y^{*}\right]}{\delta y^{*}}=\frac{\partial f}{\partial y^{*}}-\frac{d}{d x}\left(\frac{\partial f}{\partial\left(y^{*}\right)^{\prime}}\right)=0
$$

If we have constraints

$$
J_{i}[y]=\int_{a}^{b} g_{i}\left(x, y, y^{\prime}\right) d x=U_{i}(i=1,2, \ldots, M),
$$

then we solve the differential equation

$$
\frac{\delta I\left[y^{*}\right]}{\delta y^{*}}=\sum_{i=1}^{M} \lambda_{i} \frac{\delta J_{i}\left[y^{*}\right]}{\delta y^{*}}
$$

for $y^{*}(x)$ which depends on Lagrange multipliers $\lambda_{1}, \ldots, \lambda_{M}$ parametrically. When we put $y^{*}$ in the constraint equations eq.(33) we obtain a set of algebraic (in general nonlinear) sets of equation for $\lambda_{1}, \ldots, \lambda_{M}$. The solutions for $\lambda_{1}, \ldots, \lambda_{M}$ will be a function of the parameters $U_{1}, U_{2}, \ldots, U_{M}$ and when the multipliers are put in $y^{*}(x)$ back, the $y^{*}(x)$ itself will be a function of $U_{1}, U_{2}, \ldots, U_{M}$ parameters. When we feed $y^{*}(x)$ in the integral for $I[y]$ we get $I\left[y^{*}\right]$ which will be a function of $U_{1}, U_{2}, \ldots, U_{M}$ too. We denote this function as $\mathrm{I}(\mathbf{U})$ where U stands for $U_{1}, U_{2}, \ldots, U_{M}$. So we have $\mathrm{I}(\mathbf{U})=I\left[y^{*}\right]$.

Now we change the parameters $U_{1}, U_{2}, \ldots, U_{M}$ by an infinitesimal amount: $U_{i} \rightarrow U_{i}+d U_{i}$ and this changes the solution $y^{*}$ by an infinitesimal amount: $y^{*} \rightarrow y^{*}+\Delta y^{*}$ where $\Delta y^{*}$ is 
an infinitesimal function. this changes $\mathrm{I}(\mathbf{U})$ by

$$
d \mathrm{I}(\mathbf{U})=I\left[y^{*}+\Delta y^{*}\right]-I\left[y^{*}\right]=\int_{a}^{b} \frac{\delta I\left[y^{*}\right]}{\delta y}\left(\Delta y^{*}\right) d x .
$$

For the constraint we also have

$$
d U_{i}=J_{i}\left[y^{*}+\Delta y^{*}\right]-J_{i}\left[y^{*}\right]=\int_{a}^{b}\left(\delta J_{i}\left[y^{*}\right] / \delta y\right)\left(\Delta y^{*}\right) d x . \quad(i=1,2, \ldots, M) .
$$

Putting the $\delta I\left[y^{*}\right] / \delta y$ from eq.(34) into the eq.(35) and comparing to the eq.(36) we obtain

$$
d \mathrm{I}(\mathbf{U})=\lambda_{1} d U_{1}+\lambda_{2} d U_{2}+\ldots, \lambda_{M} d U_{M},
$$

which tells us that

$$
\lambda_{i}=\partial \mathrm{I}\left(U_{1}, U_{2}, \ldots, U_{M}\right) / \partial U_{i}(i=1,2, \ldots, M) .
$$

Usually both $\mathrm{I}(\mathbf{U})$ and $\mathbf{U}$ have physical meaning and this prescription assigns a physical meaning to the Lagrange multipliers $\lambda_{1}, \ldots, \lambda_{M}$.

\section{A. Example1. Hanging chain}

In mechanics Lagrange multipliers are used in many places. One kind of application involves constrained motion in Lagrangian formulation of mechanics. [13] In this application Lagrange multipliers are time dependent and it is well known that they are equal to constraint forces. The author does not see how our rule applies to this case if it does. But it is possible to find mechanics problems where the rule applicable. Here we give an example to this.

Suppose a chain of fixed length $L$ and density $\rho$ is hanging from two points $( \pm a, 0)$ in the $x-y$ plane. The shape of the chain $y(x)$ will be such that the potential energy integral

$$
V[y]=\rho g \int_{-a}^{a} y \sqrt{1+\left(y^{\prime}\right)^{2}} d x
$$

will be a minimum under the constraint that the length of the chain is fixed:

$$
\int_{-a}^{a} \sqrt{1+\left(y^{\prime}\right)^{2}} d x=L
$$


Setting up the Euler equation with a Lagrange multiplier is straightforward. Solving the arising differential equation and imposing the boundary conditions $y( \pm a)=0$ we obtain the solution

$$
\begin{aligned}
y^{*}(x) & =\frac{1}{\alpha}[\cosh (\alpha x)-\cosh (\alpha a)], \\
\lambda & =-\frac{\rho g}{\alpha} \cosh (\alpha a),
\end{aligned}
$$

where $\alpha$ is an integration constant. To obtain a relation between $\alpha$ and $L$ we put $y^{*}(x)$ in the eq.(40) to obtain

$$
\frac{2}{\alpha} \sinh (\alpha a)=L
$$

We also calculate $V\left[y^{*}\right]$ by putting the $y^{*}$ in the eq.(39) to obtain

$$
V\left[y^{*}\right]=\rho g\left(\frac{a}{\alpha}-\frac{\sinh (2 \alpha a)}{\alpha^{2}}\right) .
$$

To show that $d V\left[y^{*}\right] / d L=\lambda$ we write $d V\left[y^{*}\right] / d L$ as

$$
\frac{d V\left[y^{*}\right]}{d L}=\frac{d V\left[y^{*}\right] / d \alpha}{d L / d \alpha}
$$

and calculate $d L / d \alpha$ and $d V\left[y^{*}\right] / d \alpha$ from eqs. (43,44) to obtain

$$
\frac{d V\left[y^{*}\right]}{d L}=-\frac{\rho g}{\alpha} \cosh (\alpha a)=\lambda
$$

We showed that $d V\left[y^{*}\right] / d L=\lambda$ holds.

Now what is the physical meaning of the Lagrange multiplier $\lambda$ ? Imagine pulling the chain from one end by an infinitesimal amount $d L$. The work done is $T \cdot d L$ where $T$ is the tension at the endpoints and this should be equal to the potential energy difference $V\left[y^{*}(L-d L)\right]-V\left[y^{*}(L)\right]=-d V\left[y^{*}\right]$. Therefore $T=-d V\left[y^{*}\right] / d L=-\lambda$ and we have the physical interpretation that $-\lambda$ is equal to the tension at the ends of the chain. As usually the case in mechanics, here too the multiplier turns out to be a constraint force.

Here application of the rule $d V\left[y^{*}\right] / d L=\lambda$ makes the interpretation of multiplier a simple one-line argument. I invite the reader to try to find another argument as short as this one for the physical meaning of the multiplier without using the rule. 


\section{B. Example2. Constrained brachistochrone problem}

Here we present a mechanics problem that the multiplier is not a constraint force and yet has a very precise meaning. We have a rail that extends from point $A(x=0, y=h)$ to the point $B(x=a, y=0)$ and the rail has a fixed length $L \geq \sqrt{a^{2}+h^{2}}$. A mass slides on it from point A to point B. What is the curve that delivers the shortest travel time?

Here the travel time

$$
T[y]=\sqrt{\frac{1}{2 g}} \int_{0}^{a} \sqrt{\frac{1+(d y / d x)^{2}}{h-y}} d x,
$$

is minimized with the constraint

$$
U[y]=\int_{0}^{a} \sqrt{1+(d y / d x)^{2}} d x=L .
$$

The Euler equation

$$
\frac{\delta T[y]}{\delta y}=\lambda \frac{\delta U[y]}{\delta y},
$$

yields (after integrating once) the differential equation

$$
C \sqrt{1+(d y / d x)^{2}}=\frac{1}{\sqrt{2 g(h-y)}}-\lambda
$$

where $C$ is an integration constant. Because of difficulties with evaluating some integrals we were not able to solve this problem in closed form. Therefore we do not give a explicit demonstration that the rule $\lambda=d T\left[y^{*}\right] / d L$ holds. But assuming that the multiplier is calculated (numerically or using some approximation) we can tell physical meaning of it. It is rate of increase of optimal travel time with increasing length L. Physically we expect that as $L$ starts increasing from the initial value $\sqrt{a^{2}+h^{2}}$ the travel time $T\left[y^{*}\right]$ should decrease (hence negative $\lambda$ ) until an optimal length $L_{O}$ is reached. When $L=L_{O}$ we have $\lambda=0$ and problem reduces to unconstrained brachistochrone problem and travel time is the absolute minimum. As $L$ is increased further the travel time should increasing again yielding positive $\lambda$. Here we see that, using both our knowledge of physics and the rule enables us to find the sign of $\lambda$ without solving the problem. 


\section{Example3. Minimum uncertainty wave packet}

In some quantum problems the Lagrange multipliers have no apparent physical meanings. These cases arises especially for normalization and overlap integral constraints. Here we give an example that has two Lagrange multipliers. One of the Lagrange multipliers has a clear physical meaning obeys the rule and the other does not.

We want to find the wave packet with the minimum momentum uncertainty

$$
M[\psi]=\int_{-\infty}^{\infty} \psi^{*} \widehat{P}^{2} \psi d x-\left(\int_{-\infty}^{\infty} \psi^{*} \widehat{P} \psi d x\right)^{2}=\sigma_{P}^{2}
$$

with the conditions that position uncertainty is fixed

$$
K[\psi]=\int_{-\infty}^{\infty} \psi^{*} x^{2} \psi d x-\left(\int_{-\infty}^{\infty} \psi^{*} x \psi d x\right)^{2}=\sigma_{x}^{2}
$$

and the wave function is normalized

$$
N[\psi]=\int_{-\infty}^{\infty} \psi^{*} \psi d x=u
$$

Here $\widehat{P}=-i \hbar d / d x$ momentum operator and for the sake of the argument we consider normalization integral with a general fixed value $u$. We later take $u=1$ after showing that $u \neq 1$ has no solution. Treating $\psi$ and $\psi^{*}$ as independent variables the Euler equation with two Lagrange multipliers

$$
\frac{\delta M[\psi]}{\delta \psi^{*}}=\lambda_{1} \frac{\delta K[\psi]}{\delta \psi^{*}}+\lambda_{2} \frac{\delta N[\psi]}{\delta \psi^{*}}
$$

yields the equation

$$
-\hbar^{2} \psi^{\prime \prime}(x)+2 i \hbar b \psi^{\prime}(x)-\lambda_{1}\left(x^{2}-2 a x\right) \psi(x)-\lambda_{2} \psi(x)=0
$$

where $a$ and $b$ are the parameters defined as

$$
\begin{aligned}
& a=\int_{-\infty}^{\infty} \psi(x)^{*} x \psi(x) d x \\
& b=\int_{-\infty}^{\infty} \psi^{*}(x) \widehat{P} \psi(x) d x .
\end{aligned}
$$


As a consistency condition the solution of eq.(55) should satisfy eqs.(5657). Making the transformation $\psi(x)=e^{i b x / \hbar} \varphi(x)$ we obtain the equation

$$
-\hbar^{2} \varphi^{\prime \prime}(x)-\lambda_{1}(x-a)^{2} \varphi(x)=\left(\lambda_{2}+b^{2}-\lambda_{1} a^{2}\right) \varphi
$$

This is the Schrodinger equation for a Harmonic oscillator problem $(m=1 / 2$ and $w=$ $\sqrt{-4 \lambda_{1}}$ ) and normalizable solutions are possible only for $\lambda_{1}<0$. The solutions are the harmonic oscillator eigenfunctions $\phi_{0}, \phi_{1}, \ldots, \phi_{n}, \ldots$ where $\phi_{n}(x)$ is normalized in the usual way: $\int\left|\phi_{n}(x)\right|^{2} d x=1$. But the $\psi(x)$ must satisfy the normalization condition given in eq. (153). Therefore we take $\psi_{n}(x)=\sqrt{u} e^{i b x / \hbar} \phi_{n}(x)$. With this $\psi(x)$ we get the following expectation values

$$
\begin{aligned}
& \int_{-\infty}^{\infty} \psi_{n}^{*}(x) x \psi_{n}(x) d x=u a, \\
& \int_{-\infty}^{\infty} \psi_{n}^{*}(x) \widehat{P} \psi_{n}(x) d x=u b .
\end{aligned}
$$

The solution does not satisfy the consistency conditions given in eqs.(56 57). Therefore consistent solutions are possible only for $u=1$. We take $u=1$ for the rest of the discussion.

All $\psi_{n}(x)$ are stationary solutions and they all have $\langle\widehat{P}\rangle=b$. For minimum momentum uncertainty we must have minimum $\left\langle\widehat{P}^{2}\right\rangle$. The $\left\langle\psi_{n}\left|\widehat{P}^{2}\right| \psi_{n}\right\rangle$ is easily calculated as $b^{2}+(n+$ $1 / 2) \hbar w / 2$. Therefore for minimum momentum uncertainty we must take the ground state:

$$
\phi(x)=\left(\frac{2 k}{\pi}\right)^{1 / 4} e^{-k(x-a)^{2}},
$$

where $\langle x\rangle=a$. Putting this back into the eq.(58) yields

$$
\begin{aligned}
\lambda_{1} & =-4 k^{2} \hbar^{2} \\
2 k \hbar^{2} & =\lambda_{2}+b^{2}-\lambda_{1} a^{2} .
\end{aligned}
$$

We also calculate $\left\langle x^{2}\right\rangle$ and $\left\langle\widehat{P}^{2}\right\rangle$ for $\psi_{0}(x)$ as

$$
\begin{aligned}
& \left\langle x^{2}\right\rangle=a^{2}+1 /(4 k) \\
& \left\langle\widehat{P}^{2}\right\rangle=b^{2}+\hbar^{2} k .
\end{aligned}
$$


From this $\sigma_{x}^{2}=\left\langle x^{2}\right\rangle-\langle x\rangle^{2}=1 /(4 k)$ and $\left(\sigma_{P}^{2}\right)_{\min }=\left\langle\widehat{P}^{2}\right\rangle-\langle\widehat{P}\rangle^{2}=\hbar^{2} k$ are obtained. This yields the uncertainty principle $\left(\sigma_{P}^{2}\right)_{\min } \sigma_{x}^{2}=\hbar^{2} / 4$. The Lagrange multiplier $\lambda_{1}$ has the value $\lambda_{1}=-\hbar^{2} / 4\left(\sigma_{x}^{2}\right)^{2}$ and satisfies the rule

$$
\lambda_{1}=\frac{d\left(\sigma_{P}^{2}\right)_{\min }}{d \sigma_{x}^{2}}=-\frac{\hbar^{2}}{4\left(\sigma_{x}^{2}\right)^{2}}=-\frac{\left(\sigma_{P}^{2}\right)_{\min }}{\sigma_{x}^{2}} .
$$

The physical meaning of the lagrange multiplier is the rate of change of minimum momentum uncertainty with position uncertainty which is equal to negative ratio of both uncertainties.

The Lagrange multiplier $\lambda_{2}$ has the value $2 k \hbar^{2}-b^{2}+\lambda_{1} a^{2}$ and it has no obvious physical interpretation. We cannot apply the rule $\lambda_{2}=d\left(\sigma_{P}^{2}\right)_{\min } / d u$ either because we showed that the solution does not exist for $u \neq 1$. Even if it existed it would still be difficult to attach a meaning to it because the for $u \neq 1$ we have no physical meaning to assign to the variable $u$.

\section{References}

[1] J. W. Lorimer, 'Elementary statistical mechanics without Lagrange multiplier', J. Chem. Education 43, 39-40 (1966)

[2] D. Kleppner, 'Avoiding Lagrange multipliers in introductory statistical mechanics', Am. J. Phys. 36, 843 (1968)

[3] D. Kleppner, 'Avoiding Lagrange multipliers in introductory statistical mechanics', Am. J. Phys. 37, 457 (1969)

[4] R. D. Turoff, 'Avoiding Stirling's approximation and Lagrange multipliers in introductory statistical mechanics',Am. J. Phys. 38, 387-389 (1970)

[5] R. D. Turoff, 'Correction to 'Avoiding Stirling's approximation and Lagrange multipliers in introductory statistical mechanics",Am. J. Phys. 39, 580-582 (1971)

[6] A. C. Chiang, Fundamental Methods of Mathematical Economics (McGraw-Hill company, 1974), 2nd ed., pp. 380-381 
[7] J. V. Baxley, J. C. Moohouse, 'Lagrange multiplier problems in economics', The Am. Math. Monthly, 91, (7), 404-412 (1984)

[8] D. P. Bertsekas, Constrained Optimization and Lagrange Multiplier Methods (Academic Press, 1982) pp. 70

[9] D. R. LaTorre, J. W. Kenelly, I. B. Fetta, L. L. Carpenter, C. R. Harris, Calculus Concepts: an Informal Approach to Mathematics of Change (Boston, MA: Houghton Mifflin Company, 1998) pp. 650-651

[10] G. C. Berresford, A. M. Rockett, Applied Calculus (Boston, MA: Houghton Mifflin Company, 2000) pp. 568-569, 584-585

[11] F. Y. M. Wan, Introduction to Calculus of Variations and its Applications (Chapman\&Hall, 1995), pp. 300-301

[12] K. Huang, Statistical Mechanics (John Wiley\&Sons, 1987), 2nd ed., pp. 179-185

[13] H. Goldstein, C. Poole, J. Safko, Classical Mechanics (Pearson- Addison Wesley 2002) 3rd edn pp 45-50 